June 1993

HU Berlin-IEP-93/2

\title{
Dirac sheets and gauge fixing in $U(1)$ lattice gauge theory 1
}

\author{
V.G. Bornyakov ${ }^{1}$, V.K. Mitrjushkin ${ }^{2}$ 直, M. Müller-Preussker ${ }^{2}$, \\ F. Pahl ${ }^{3}$, \\ ${ }^{1}$ Institute of High Energy Physics, Protvino, Russia \\ ${ }^{2}$ Humboldt-Universität, Fachbereich Physik, Berlin, Germany \\ ${ }^{3}$ Freie Universität, Fachbereich Physik, Berlin, Germany
}

\begin{abstract}
Photon correlators in $U(1)$ pure gauge theory for different lattice actions are considered under the Lorentz gauge condition. They are shown to depend strongly on the gauge fixing ambiguity and on the corresponding existence of Dirac sheets. For the Coulomb phase a gauge fixing algorithm is proposed which avoids Dirac sheets and allows to find the global extremum of the non-local gauge condition.
\end{abstract}

\footnotetext{
${ }^{\dagger}$ Work supported by the Deutsche Forschungsgemeinschaft under research grant Mu 932/1-1

${ }^{\ddagger}$ Permanent address: Joint Institute for Nuclear Research, Dubna, Russia
} 


\section{Introduction}

Gauge fixing is needed in manifestly gauge invariant lattice gauge theories for nonperturbative calculations of gauge dependent quantities such as fermionic and gauge field propagators. It became a useful tool for investigations of the vacuum structure in non-Abelian lattice gauge theories, too.

However, one is faced with the non-uniqueness of the solutions of the commonly used gauge conditions, Lorentz, Coulomb gauge, etc. [1]. Let us call the different solutions of the same differential gauge condition gauge copies. In practice, the existence of different gauge copies can strongly affect the numerical computation of gauge dependent quantities.

For $U(1)$ lattice theory the existence of gauge copies has been well established (see papers [2 - 6]). It should be mentioned that the ambiguity also exists for the continuum $U(1)$ gauge theory, if the latter is treated on a four-torus [7]].

The existence of gauge copies raises the following questions.

- Are there copies due to pure lattice artifacts?

- What is the influence of gauge copies on physical quantities such as field propagators?

- How should a unique gauge fixing prescription look like?

It is the goal of this paper to provide answers for four-dimensional compact $U(1)$ lattice gauge theory with periodic boundary conditions and within the Coulomb phase.

Let us consider (lattice) gauge conditions of the type

$$
\sum_{\mu=1}^{n} \partial_{\mu}^{(-)} \operatorname{Im} U_{x \mu}=\sum_{\mu=1}^{n} \partial_{\mu}^{(-)} \sin \theta_{x \mu}=0
$$

where $U_{x \mu} \equiv \exp \left(i \theta_{x \mu}\right)$ and the lattice derivative $\partial_{\mu}^{(-)} f_{x} \equiv f_{x}-f_{x-\hat{\mu}}$. The case $n=4$ represents the nonlinear (lattice) Lorentz gauge condition [8] - often called also Landau gauge -, whereas $n=3$ leads to the Coulomb gauge. The differential gauge conditions are equivalent to finding stationary points of the functional $F$

$$
F(\theta)=\frac{1}{V} \sum_{x} F_{x}(\theta), \quad F_{x}(\theta)=\frac{1}{2 n} \sum_{\mu=1}^{n}\left[\cos \theta_{x \mu}+\cos \theta_{x-\hat{\mu} ; \mu}\right]
$$

with respect to periodic gauge transformations ( $V$ denotes the number of lattice sites). Obviously, the requirement of a global maximization of expression (2) is stronger than the differential condition (11).

The usual way to fix the gauge is as follows [8]. The equilibrium configurations produced by Monte Carlo updating are independently gauge transformed in order to satisfy the gauge condition. In case of the gauge conditions (1) this is performed 
by means of an iterative procedure which maximizes $F_{x}(\theta)$ site by site. We shall call this algorithm standard gauge cooling. In order to improve convergence overrelaxation steps are usually built in. One can easily convince oneself numerically that standard gauge cooling for Lorentz and Coulomb gauge leads to various stable local maxima related to different $F$ values. The following discussions will mainly apply to the Lorentz gauge.

In a recent paper [6], it was shown that in the Coulomb phase some of the gauge copies produce a photon propagator with a decay behaviour inconsistent with the expected zero mass behaviour. The origin of these 'pathological' gauge copies was not clarified, but a recipe to avoid this problem was proposed. According to this, one has to generate several gauge copies by applying standard gauge cooling to randomly gauge transformed equilibrium configurations. Then choosing the gauge copies with maximal $F(\theta)$ values significantly improves the photon mass estimate [6].

In the following we want to go a step further. We are going to show that the 'bad' gauge copies are related to the existence of pairs of Dirac sheets, and we propose an algorithm which removes them in a straightforward way.

\section{$2 \quad$ Lattice actions}

We consider $U(1)$ lattice gauge theory on the torus $T^{4}$. In our calculations, we first apply the standard Wilson action (WA)

$$
S_{W}\left(U_{l}\right)=\beta \cdot \sum_{P}\left(1-\cos \theta_{P}\right)
$$

where plaquette angles $\theta_{P} \equiv \theta_{x, \mu \nu}$ are given by $\theta_{x, \mu \nu}=\theta_{x \mu}+\theta_{x+\hat{\mu}, \nu}-\theta_{x+\hat{\nu}, \mu}-\theta_{x \nu}$. The plaquette angle $\theta_{P}$ can be split up [9]

$$
\theta_{P}=\bar{\theta}_{P}+2 \pi n_{P}, \quad-\pi<\bar{\theta}_{P} \leq \pi, \quad n_{P}=0, \pm 1, \pm 2
$$

where the gauge invariant part of the plaquette angle, $\bar{\theta}_{P}$, describes the electromagnetic flux through the plaquette, and $n_{P}$ is the number of Dirac strings passing through it. Let us call plaquettes with $n_{P} \neq 0$ Dirac plaquettes (DP).

Monopole charges $K_{c}$ in elementary 3D-cubes $c$ are then defined by the net number of strings entering or exiting these cubes. The worldlines of these Abelian monopoles on the dual lattice close either within the lattice volume or by periodic boundary conditions. The dual integer valued plaquettes

$$
m_{x, \mu \nu}=\frac{1}{2} \varepsilon_{\mu \nu \rho \sigma} n_{x, \rho \sigma}
$$

form Dirac sheets bounded by the worldlines of monopole-antimonopole pairs or closed within the lattice volume or due to periodic boundary conditions. 
In a recent paper [10], we showed that the monopoles (as well as negative plaquette values) have to be understood as lattice artifacts. Their suppression removes the phase transition of the standard Wilson theory at $\beta \simeq 1$ and as a consequence provides a uniform Coulomb phase in the whole positive $\beta$ range (see also [11]). Therefore, we will discuss also the modified compact action (MA):

$$
S_{M A}=S_{W}+\lambda_{K} \cdot \sum_{c}\left|K_{c}\right|+\lambda_{Z} \cdot \sum_{P}\left(1-\operatorname{sign}\left(\cos \theta_{P}\right)\right), \quad \lambda_{K}, \lambda_{Z}=\infty .
$$

Note that this modification does not influence the classical continuum limit and does not change any perturbative aspects. It may serve as an alternative for nonperturbative QED investigations carried out so far with the non-compact gauge action.

\section{Gauge fixing and Dirac sheets}

Most of our calculations were done on $6^{4}$ and $12 \cdot 6^{3}$ lattices, for some exploratory calculations also on $20^{4}$ and $28 \cdot 14^{3}$ lattices, respectively.

First we applied ten random gauge transformations to each Monte Carlo equilibrium configuration. On each of these versions, we carried out standard gauge cooling with the stopping criterion

$$
\operatorname{Max}\left|\sum_{\mu} \partial_{\mu}^{(-)} \sin \theta_{x \mu}\right|<10^{-5} \text { and } \frac{1}{\mathrm{~V}} \sum_{x}\left|\sum_{\mu} \partial_{\mu}^{(-)} \sin \theta_{x \mu}\right|<10^{-6} .
$$

We determined the average number $\left\langle N_{l m}\right\rangle$ of different local maxima $F_{\max }(\theta)$ of the functional (2) found for each equilibrium configuration. Numerically, two local maxima are understood to be identical if the difference of corresponding $F_{\max }(\theta)$ values is less than $\epsilon=10^{-5}$. (To make sure that this recipe is well-defined, we varied the value of $\epsilon$ and found that for $\epsilon=10^{-4}$ and $10^{-5}$, the change in $\left\langle N_{l m}\right\rangle$ was less than the statistical errors.) For the Wilson action in the confinement phase, we found $\left\langle N_{l m}\right\rangle \simeq 10$; i. e. practically all gauge copies produced are related to distinct values $F_{\max }(\theta)$. At $\beta \sim 1$, this average drops sharply down to $\left\langle N_{l m}\right\rangle \simeq 3$ and approximately does not depend on $\beta$ within the Coulomb phase. For the modified action, the value of $\left\langle N_{l m}\right\rangle$ is seen to be independent of the coupling in the whole interval of $\beta$ 's considered and coincides with that for the Wilson action in the Coulomb phase.

The functional dependence of $\left\langle N_{l m}\right\rangle=f(\beta)$ for both actions turned out to look very similar to the behaviour of the average number of Dirac plaquettes $\left\langle N_{D P}\right\rangle=g(\beta)$ after standard gauge cooling. Therefore, we guess that both the values of $F_{\max }(\theta)$ and the number of different local maxima are correlated with $\left\langle N_{D P}\right\rangle$.

Another observation made in both the Coulomb and the confinement phase (for Wilson action) proves this idea. There is a strong correlation of the local quantity 
$F_{x}(\theta)$ with the number of Dirac plaquettes among the 24 plaquettes sharing the site $x$.

By a more detailed investigation of the number and the location of Dirac plaquettes in the Coulomb phase, we observed pairs of Dirac sheets closing by periodic boundary conditions. In the extreme case of a minimal surface this is a pair of periodic Dirac sheets occupying parallel planes and having corresponding Dirac plaquettes with opposite signs. In general the surfaces fluctuate around this extreme case. The minimal number of Dirac plaquettes in such a lattice gauge field is equal to $2 \cdot L_{\mu} \cdot L_{\nu}$, where $L_{\mu}, L_{\nu}, \mu, \nu=1, \ldots, 4$ denote the corresponding linear extensions of the lattice.

In contrast to monopoles, gauge transformations can move and deform Dirac sheets. Therefore, we should be able to remove double periodic Dirac sheets - called below simply double Dirac sheets (DDS) - by gauge transformations. However, it is worth noting that this property is in contrast to that of single periodic Dirac sheets as investigated e.g. in [12].

In the following we shall discuss gauge fixing with a special focus on the role of DDS. To each equilibrium configuration obtained originally from a cold start, we apply the standard gauge cooling procedure only once. Fig. 1a shows the resulting distribution $P\left(N_{D P}\right)$ for the modified action on a $12 \cdot 6^{3}$ lattice for $\beta=1.1$. There are only lattice configurations either with $N_{D P}=0$ or $N_{D P} \geq 2 \cdot 6^{2}=72$, the latter attributed to DDS. For the standard Wilson action at $\beta=1.1$ the corresponding distribution looks less pronounced because of monopoles, which are still allowed in the Coulomb phase. But in this case, another plot makes the situation transparent again. On each configuration obtained after gauge fixing, we measure the number of Dirac plaquettes separately for every twodimensional plane orientation and select the one with the maximal number $N_{D P}^{\max }$. Fig. 1b shows the corresponding distribution for the $N_{D P}^{\max }$ values. There are two nice distinct peaks in the distribution - related to configurations either with or without DDS.

There are no DDS (or even single ones) in the $\mu-\nu$-plane provided $N_{D P}^{\max }<$ $L_{\mu} \cdot L_{\nu}$ is satisfied. We can use this criterion for dividing the gauge copies into two distinct classes: those which definitely do not contain DDS and those where DDS cannot be excluded. Fig. 2 shows a scatter plot for the local maximum values $F_{\max }(\theta)$ found for 1000 equilibrium configurations after standard gauge cooling for the Wilson action (WA) at $\beta=1.1$. One can see that the crosses (copies without DDS) lie systematically higher than the full squares (copies presumably with DDS). The corresponding average values are indicated by broken lines. For the modified action (MA) at the same $\beta$, the analogous plot looks similar, but with the averages shifted to larger $F_{\max }$ values. 


\section{The photon correlator}

Let us now consider the (lattice) photon propagator. The corresponding correlation functions $\Gamma(\tau ; \vec{p})$ are defined by

$$
\Gamma(\tau ; \vec{p})=\left\langle\Phi^{*}(t+\tau ; \vec{p}) \cdot \Phi(t ; \vec{p})\right\rangle, \quad \Phi(t ; \vec{p})=\sum_{\vec{x}} e^{-i \vec{x} \vec{p}} \cdot \Phi(t ; \vec{x})
$$

with

- the gauge dependent link operator

$$
\Phi(t ; \vec{x})=\sin \theta_{x i}, \quad i=1,2,3
$$

or

- the gauge invariant plaquette operator for the $1^{+-}$state

$$
\Phi(t ; \vec{x})=\sin \theta_{x, i j}, \quad i, j=1,2,3
$$

For non-vanishing momentum the latter state has an overlap with the $1^{--}$ photon state [13].

For the gauge invariant operator, the correlator behaves as

$$
\frac{\Gamma(\tau ; \vec{p})}{\Gamma(0 ; \vec{p})}=A \cdot\left[\exp (-\tau \cdot E(\vec{p}))+\exp \left(-\left(L_{4}-\tau\right) \cdot E(\vec{p})\right)\right]+\ldots, \quad 0 \leq A \leq 1
$$

where dots correspond to higher state contributions. In the massless case, the energy $E(\vec{p})$ is given by the lattice dispersion relation:

$$
\sinh ^{2} \frac{E}{2}=\sum_{i=1}^{3} \sin ^{2} \frac{p_{i}}{2} .
$$

Provided the Lorentz gauge is properly fixed, the transverse photon propagator in the Coulomb phase is expected to have the same simple form eq. (11). We used the momenta $\vec{p}=\frac{2 \pi}{6} \cdot(0 ; k ; 0), k=1,2$ in computing the correlators and accordingly the gauge dependent operator (9) with $i=1,3$ as well as the gauge invariant one (10) with $(i, j)=(1,2),(2,3)$.

Our results for all the correlators are plotted in Fig. 3a in case of the Wilson action at $\beta=1.1$, i. e. in the Coulomb phase. One can see that the propagator obtained from operator (9) and averaged over all configurations without taking care of the presence of DDS (crosses) clearly deviates from the theoretical curve, eqs. (11, 12). Full circles correspond to an average over such gauge copies which presumably possess DDS. The deviation from the theoretical curve becomes even more pronounced in this case. But the correlator obtained after averaging only over those gauge copies which definitely do not contain DDS nicely agrees with 
the theoretical expectation. We show also the correlator for the gauge invariant operator eq. (10) (squares). For the Wilson action its points lie somewhat below the theoretical curve. This is a known effect [10 which is due to the fact that the overlap coefficient $A$ for the plaquette operator is less than one, i.e. there is a non-negligible admixture of higher states.

Fig. $3 \mathrm{~b}$ shows the analogous results for the modified action at $\beta=0.9$. Here we obtain a perfect agreement between the photon correlator measured with both the operators (9) - if configurations with DDS are excluded - and (10) and the theoretically expected curve.

An analogous investigation for the transverse photon correlator in the Coulomb gauge produced similar results.

Thus, we conclude that the presence of DDS as gauge and lattice artifacts spoils the correct determination of the photon mass from the gauge dependent operator (9).

\section{Improved gauge fixing algorithm}

A Polyakov loop investigation will be helpful to propose a gauge fixing algorithm allowing to remove DDS.

For a given configuration on a lattice with linear extensions $L_{\mu}, \quad \mu=1, \ldots, 4$, we define the average Polyakov loop $\overline{P_{\mu}}$ along direction $\mu$

$$
\overline{P_{\mu}}=\frac{1}{V_{\mu}} \sum_{\vec{x}} \prod_{x_{\mu}} U_{x \mu} \equiv\left|\overline{P_{\mu}}\right| \cdot \mathrm{e}^{i \psi_{\mu}}, \quad-\pi<\psi_{\mu} \leq \pi,
$$

where $V_{\mu}=V / L_{\mu}$. In Fig. 4 for the Wilson action at $\beta=1.1$, we show the scatter plot of $\overline{P_{\mu}}$ values in the complex plane for 400 Monte Carlo equilibrium configurations investigated after standard gauge cooling. The distribution has the form of a circle, as it should be. But the points corresponding to DDS orthogonal to the $\mu$-direction are not distributed with rotational symmetry. They occupy only the left part of the circle, i.e. $\operatorname{Re}\left(\overline{P_{\mu}}\right)<0$.

It is worth noting that gauge transformations which are implemented in the standard gauge cooling procedure are periodic by construction. Each local $P_{\mu}$ value cannot change under standard gauge cooling transformations, and therefore, their distribution is also invariant.

This suggests how to avoid gauge copies with DDS. We carry out nonperiodic gauge transformations

$$
U_{x \mu} \rightarrow g_{x}^{*} \cdot U_{x \mu} \cdot g_{x+\hat{\mu}}=U_{x \mu} \cdot e^{i c_{\mu}}, \quad g_{x}=e^{i c_{\mu} x_{\mu}}
$$

or equivalently

$$
\theta_{x \mu} \rightarrow \theta_{x \mu}+c_{\mu}
$$


which turn all $\overline{P_{\mu}}, \quad \mu=1, \ldots, 4$ into real positive numbers before applying standard gauge cooling. Therefore, we put

$$
c_{\mu}=-\psi_{\mu} / L_{\mu}, \quad \mu=1, \ldots, 4
$$

As is evident from Fig. 4, there are no gauge copies with DDS among configurations with large positive real part of $\overline{P_{\mu}}$. This gives us confidence that after a gauge transformation of the type (14), which we will call preconditioning step, subsequent gauge cooling should avoid local maxima with DDS. Our computations (with around 30000 measurements for the same $\beta$ and action as in Fig. 4) confirmed this conjecture. No gauge copies with DDS were found.

The reader will be convinced also by Fig. 5. Starting for every equilibrium configuration with the preconditioning step, we look after each subsequent gauge cooling sweep for the layer with the maximal number of Dirac plaquettes $N_{D P}^{\max }$ as explained above. $N_{D P}^{\max }$ has been plotted for all 400 configurations investigated as a function of the number of gauge cooling sweeps $N_{g s w}$. On a $12 \cdot 6^{3}$ lattice, already after 12 sweeps no configurations with $N_{D P}^{\max } \geq 36$ were left. We checked the algorithm described above also with larger lattices $\left(14^{3} \cdot 28\right.$ and $20^{4}$, respectively) in the Coulomb phase. There were no DDS.

It is important to stress that the above procedure avoiding DDS already allows us to reproduce the correct photon propagator despite the fact that it does not yet provide unique gauge fixing. The global maximum is not really necessary for solving the photon mass problem.

However, it appears that a combination of nonperiodic gauge transformations (14) and gauge cooling permits to find the global maximum of $F$. The recipe looks as follows. After the preconditioning step, we carry out gauge cooling sweeps until DDS are removed (around 10 maximization and overrelaxation sweeps for the lattice sizes discussed). In a third step, we maximize $F(\theta)$ with respect to nonperiodic gauge transformations (14). Thus, the global phase shifts $c_{\mu}, \mu=1, \ldots, 4$ are fixed such that

$$
F_{\mu}(\theta) \equiv \frac{1}{4 \mathrm{~V}} \sum_{x} U_{x \mu} \equiv\left|F_{\mu}\right| \cdot \mathrm{e}^{i \chi_{\mu}} \rightarrow\left|F_{\mu}\right|, \quad \mu=1, \ldots, 4
$$

become real and positive. Since this maximization generally violates the local gauge condition (1) we mix it with further gauge cooling iterations. This procedure can be repeated until the changes in $F(\theta)$ during the nonperiodic gauge transformations vanish within machine precision. We checked the whole recipe by applying it again to 10 randomly gauge transformed versions of each Monte Carlo equilibrium field. Only for $0.5 \%$ of all equilibrium configurations we still found gauge copies. They are, however, related to each other by gauge transformations $g_{x}$ significantly differing from unity only in a very narrow neighbourhood of a single site!

The algorithm proposed here applies also to the Coulomb gauge in an analogous manner. 


\section{Conclusions}

Let us summarize our findings.

- We have convinced ourselves that gauge copies which possess double Dirac sheets (DDS) give rise to a wrong behaviour of the transverse photon propagator in the Coulomb phase.

- The transverse photon propagator obtained by averaging over gauge copies without DDS shows an agreement with the expected theoretical curve which for the standard Wilson action near to the deconfinement phase transition is even better than for the gauge invariant plaquette correlator.

- We proposed and checked an algorithm for Lorentz (and Coulomb) gauge fixing which avoids gauge copies with DDS.

- Nonperiodic gauge transformations were shown to allow unique gauge fixing to the global extremum of the gauge functional $F(\theta)$ in almost all cases. This might be of importance for fermion propagator investigations now under way.

We did not consider the confinement phase in case of the standard Wilson action. Because of the existence of monopole excitations, a unique gauge fixing is more involved than for the Coulomb phase. Since this is even more important for nonAbelian gauge theories, we hope to come back to this point very soon.

\section{Acknowledgements}

One of us (V.G.B.) would like to express his deep gratitude to all colleagues at Humboldt University for hospitality.

\section{Note added in proof}

After having written up this letter, we became aware of the recent paper by M.I. Polikarpov, Ken Yee and M.A. Zubkov, preprint LSU-431-93, hep-lat/9305019, where some of the conclusions about the role of Dirac strings in lattice QED are drawn, too. 


\section{References}

[1] V.N. Gribov, Nucl. Phys. B139 (1978) 1

[2] P. Coddington, A. Hey, J. Mandula and M. Ogilvie, Phys. Lett. 197B (1987) 191

[3] A. Nakamura and R. Sinclair, Phys. Lett. 243B (1990) 396

[4] A. Hulsebos, M.L. Laursen, J. Smit and A.J. van der Sijs, Nucl. Phys. B (Proc. Suppl.) 20 (1991) 98

[5] Ph. de Forcrand, J.E. Hetrick, A. Nakamura and M. Plewnia, Nucl. Phys. B (Proc. Suppl.) 20 (1991) 194

[6] A. Nakamura and M. Plewnia, Phys. Lett. 255B (1991) 274

[7] T.P. Killingback, Phys. Lett. 138B (1984) 87

[8] J. Mandula and M. Ogilvie, Phys. Lett. 185B (1987) 127

[9] T.A. DeGrand and D. Toussaint, Phys. Rev. D22 (1980) 2478

[10] V.G. Bornyakov, V.K. Mitrjushkin and M. Müller-Preussker, Nucl. Phys. B (Proc. Suppl.) 30 (1993) 587

[11] J. S. Barber, R. E. Schrock and R. Schrader, Phys. Lett. B152 (1985) 221

J. S. Barber and R. E. Schrock, Nucl. Phys. B257 (1985) 515

[12] V. Grösch, K. Jansen, T. Jersák, C.B. Lang, T. Neuhaus and C. Rebbi, Phys. Lett. 162B (1985) 171

[13] B. Berg and C. Panagiotakopoulos, Phys. Rev. Lett. 52 (1984) 94 


\section{Figure captions}

Fig.1. Distributions of the number of Dirac plaquettes $P\left(N_{D P}\right)$ on a $12 \cdot 6^{3}$ lattice at $\beta=1.1$ for modified action (MA) (a) and for Wilson action (WA) (b). In the latter case, only those plane orientations are selected which contain the maximal number of Dirac plaquettes $N_{D P}^{\max }$.

Fig.2. $F_{\max }(\theta)$ for different configurations for WA on a $6^{4}$ lattice for $\beta=1.1$. Crosses (squares) correspond to configurations without (presumably with) double Dirac sheets. Broken lines are the corresponding averages for the two classes of configurations.

Fig.3. Photon correlators $\Gamma(\tau ; \vec{p}) / \Gamma(0 ; \vec{p})$ with momentum $\vec{p}=\frac{2 \pi}{6} \cdot(0 ; 1 ; 0)$ on $12 \cdot 6^{3}$ lattices at $\beta=1.1$ for WA (a) and at $\beta=0.9$ for MA (b). Open (full) circles correspond to transverse correlators calculated on the configurations without (with) double Dirac sheets. Crosses show the transverse correlators averaged over all configurations. Squares correspond to plaquette-plaquette correlators. The solid line represents the expected zero-mass behaviour (12).

Fig.4. Scatter plot for Polyakov loop values $\overline{P_{\mu}}, \quad \mu=1,2,3$ in the complex plane after standard gauge cooling (WA, $\beta=1.1,12 \cdot 6^{3}, 400$ measurements). Squares (crosses) correspond to the existence (non-existence) of double Dirac sheets in planes orthogonal to $\mu$-direction.

Fig.5. $\quad N_{D P}^{\max }$ versus number of gauge cooling sweeps $N_{g s w}$ after preconditioning (WA, $\beta=1.1,12 \cdot 6^{3}, 400$ measurements). 